\title{
Nonlinear rheology of hyperbranched polyisobutylene
}

\author{
C. G. Robertson ${ }^{\text {a) }}$ and C. M. Roland ${ }^{\text {b) }}$ \\ Naval Research Laboratory, Chemistry Division, Code 6120, \\ Washington, D.C. 20375-5342 \\ J. E. Puskas \\ Department of Chemical and Biochemical Engineering, University of Western \\ Ontario, London Ontario N6A 5B9, Canada
}

(Received 13 July 2001; final revision received 18 October 2001)

\begin{abstract}
Synopsis
The nonlinear shear rheology of a hyperbranched polyisobutylene (PIB) with narrow molecular weight distribution $\left(M_{w} / M_{n}=1.3\right)$ was compared to that of a polydisperse $\left(M_{w} / M_{n}=2.5\right)$ linear PIB. After adjusting the respective measurement temperatures to yield equal shear viscosities, the rheology was found to be quite similar for the two polymers, notwithstanding their markedly different structures. These similarities persisted in capillary extrusion experiments at moderate shear rates, on the compounds reinforced with carbon black. However, at higher shear rates, the linear PIB exhibits a greater elastic response, with consequently larger extrudate swell. (C) 2002 The Society of Rheology. [DOI: 10.1122/1.1428318]
\end{abstract}

\section{INTRODUCTION}

There has been renewed interest of late in the effect of long-chain branching on the rheology of polymer melts, with a particular focus on highly branched polymers. If the branches are unentangled, low viscosities can be obtained in high molecular weight materials. However, chain ends contribute to mechanical hysteresis and lower strength in crosslinked polymers [Flory (1947)]. Thus, although entangled branches increase the melt viscosity, the motivation for developing polymers with branched topologies is to improve processing through modification of shear thinning and extensional flow.

While structure-property relationships and models for the dynamics of branched polymers are usually developed from linear viscoelastic data, it is the nonlinear viscoelastic response which governs processing, and hence the practical utility of polymers. Branched polymers differ from their linear counterparts with respect to both the shear-rate dependence of viscosity [Graessley (1977); Fetters et al. (1993)] and the strain dependence of their nonlinear properties [Osaki and Kurata (1980); Bick and McLeish (1996); Kasehagen and Macosko (1998)]. Long-chain branching can induce shear thinning at lower shear rates [Kim et al. (1996); Wood-Adams (2001)], while also making the material more prone to extensional hardening [Meissner (1975); Pearson et al. (1983); Hingmann and Marczinke (1994); Inkson et al. (1999)]. At constant molecular weight, low levels of entangled branches may also increase the magnitude of the primary normal

\footnotetext{
${ }^{a)}$ Present address: ExxonMobil Chemical Company; Baytown, TX 77522-5200.

b) Author to whom all correspondence should be addressed; electronic mail: mike.roland@nrl.navy.mil
} 
TABLE I. Macrostructure of polyisobutylenes.

\begin{tabular}{cccccc}
\hline \hline Designation & Structure & $\begin{array}{c}M_{w} \times 10^{-3 \mathrm{a}} \\
(\text { daltons })\end{array}$ & $M_{w} / M_{n}{ }^{\mathrm{a}}$ & $\begin{array}{c}M_{B r} \times 10^{-3 \mathrm{~b}} \\
(\text { daltons })\end{array}$ & $f^{\mathrm{c}}$ \\
\hline L176 & linear & 176 & 1.6 & $\ldots$ & $\ldots$ \\
L302 & linear & 302 & 1.8 & $\ldots$ & $\ldots$ \\
L389 & linear & 389 & 2.5 & $\ldots$ & $\ldots$ \\
H1080_21 & hyperbranched & 1,080 & 2.6 & 52.7 & 20.5 \\
H984_57 & hyperbranched & 984 & 1.3 & 17.2 & 57.2 \\
H230_9 & hyperbranched & 230 & 2.1 & 23.5 & 9.2 \\
\hline \hline
\end{tabular}

${ }^{\mathrm{a}}$ MALLS.

${ }^{\mathrm{b}} M_{w}$ of branch.

${ }^{c}$ number average.

stress coefficient [Wood-Adams (2001)]. Generally, assessments of the effect of branching are problematic, particularly for random and highly branched polymers, since the basis for comparison to linear chains may be arbitrary.

Recently, we characterized the linear viscoelastic properties of a series of hyperbranched polyisobutylenes (PIB) [Robertson et al. (2001)]. "Hyper" simply means there are many branches. These extend between successive tiers in a random, tree-like structure ("arborescent combs"), analogous to Cayley trees [Bick and McLeish (1996); Atilgan et al. (1998)]. Although the branches are sufficiently long to be entangled, the viscosity of the hyperbranched PIB exceeds that of linear polyisobutylene only for molecular weights exceeding about half a million daltons. Interestingly, the viscosity of hyperbranched PIB was found to be governed primarily by the number of branches, rather than their length. Similarly, the length of the plateau in the dynamic storage modulus depends primarily on the branching frequency, rather than the branch length. Such behavior is quite different from that of star-branched polymers.

In the present work, we extend our study of hyperbranched PIB to address whether a high concentration of entangled branches provides unique rheological properties, not otherwise achievable with linear PIB. In particular, we compare a relatively monodisperse hyperbranched PIB to linear PIB having a broad molecular weight distribution. The basis for this comparison is the equivalence of the respective viscosities and their shear rate dependence, since these govern the efficiency of processing operations, and hence the practical appeal of a material.

\section{EXPERIMENT}

The polymers used in this work are listed in Table I. The linear PIB were obtained from Aldrich. Hyperbranched polyisobutylenes, having a range of molecular weights and branch frequencies, were synthesized by copolymerizing isobutylene and an inimer, 4-(2methoxy-isopropyl) styrene (p-methoxycumyl styrene) [Paulo and Puskas (2001)]. Molecular weights were measured by gel permeation chromatography (GPC) (Waters Corp.) coupled with multiangle laser light scattering (Wyatt Technology). The branch molecular weights were determined by GPC after selective cleavage at the aromatic ring linkages via the technique described by Kennedy et al. (1981). The molecular weight distribution after this link destruction was equal to 2; this is the most probable distribution, affirming that the branching is random. The analytical results were also in agreement with values calculated from the monomer/inimer ratios. 
The hyperbranched samples are identified herein by their weight average molecular weight and number average branch frequency $\left(f=M_{n} / M_{B r}\right.$, in which $M_{B r}$ is the number average); thus, for H984_57, $M_{w}=984000$ daltons and $f=57$ branches per molecule. For hyperbranched polymers, the identity of a branch is problematic, since some are interior while others are dangling (i.e., unrestricted on one end). The number of dangling chains per molecule, assuming trifunctional branch junctions and a regular cascading tree-like structure, is equal to $(f+3) / 2$ [Janzen and Colby (1999)].

Filled compounds were formulated with 100 parts by weight of the PIB, 50 parts N339 carbon black (ASTM D1765) and five parts paraffin oil. Initial incorporation of the ingredients was carried out in a C.W. Brabender Prep Center, followed by mixing on a two-roll mill. To indicate a filled compound as opposed to a neat polymer, we use, for example, the nomenclature H984_57-f, where f signifies a compound with carbon black and oil.

As previously detailed [Robertson et al. (2001)], a Haake RS-150 RheoStress was used to measure shear viscosities in creep. Dynamic mechanical measurements were carried out on the neat polymers in a parallel plate geometry, using a Bohlin VOR and a Rheometrics ARES instruments. For the filled rubbers, a cone and plate geometry (25 $\mathrm{mm}$ diameter and 0.1 radian cone angle) was necessary, due to the strain dependence of the carbon black structure. Shear flow experiments were also made on the neat PIB, using the ARES with the cone and plate fixtures.

An Instron Model 3211 was used for capillary rheometry. Three dies having $1.5 \mathrm{~mm}$ diameter and lengths of 25,38 , and $51 \mathrm{~mm}$ were used, yielding $L / D$ ratios from 17 to 34 . Measurements were obtained after the force became constant (i.e., fully developed flow). These data were corrected for both end effects [Bagley (1957)] and nonuniform shear rate, the latter using the Weissenberg-Rabinowitsch procedure [Carreau et al. (1997)]. The Bagley plots were sufficiently linear to allow determination of the entrance pressure drops, with the assumption this pressure loss arose predominantly from the converging flow. The capillary angle of the dies was $45^{\circ}$, between the $9.5 \mathrm{~mm}$ diameter barrel and the $1.5 \mathrm{~mm}$ diameter capillary. Nine days after the capillary experiments were performed, the extrudate swell was measured for samples extruded from the $38 \mathrm{~mm}$ die.

\section{RESULTS AND DISCUSSION}

\section{A. Neat polymers}

\section{Cox-Merz and Gleissle relations}

Previously, we described the dynamic mechanical behavior of hyperbranched PIB in the linear viscoelastic regime [Robertson et al. (2001)]. While herein we extend the characterization to large strains, it is of interest to assess whether linear viscoelastic data can be used to estimate the viscosity for steady shearing flows. Strains for the latter are an order of magnitude larger than for oscillatory experiments. The dynamic viscosity is given by [Ferry (1980)]

$$
\eta^{*}(\omega)=\frac{G^{*}(\omega)}{\omega},
$$

and, according to Cox and Merz (1958), its magnitude is equivalent to the viscosity measured for steady state shear flow

$$
\left|\eta^{*}(\omega)\right|_{\dot{\gamma}=\omega}=\eta_{s s}(\dot{\gamma}),
$$




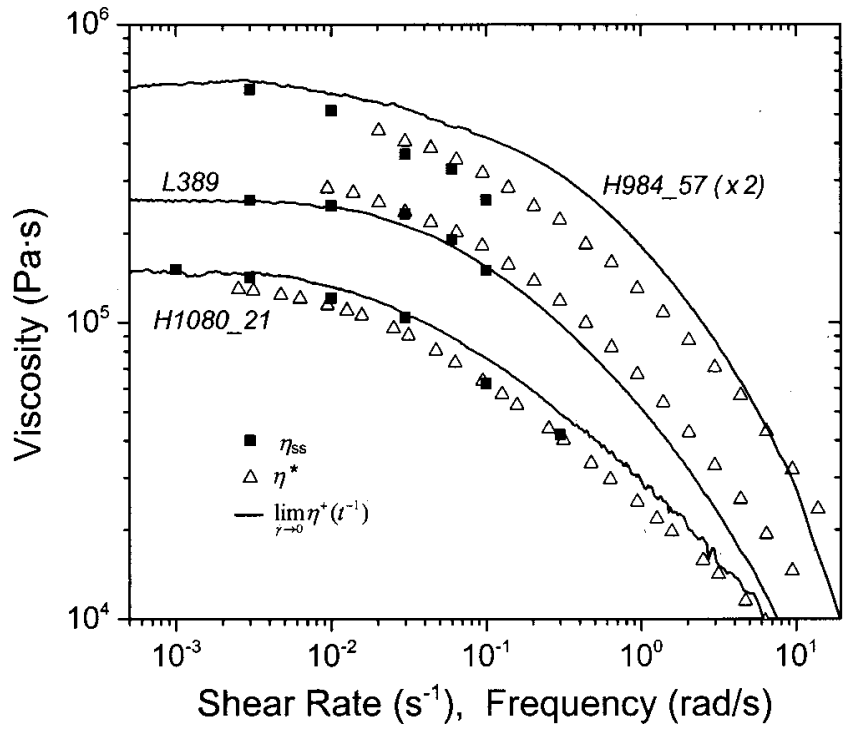

FIG. 1. Comparison of the steady-state shear viscosity (ם), the dynamic viscosity $(\triangle)$, and the viscosity calculated from the shear stress growth using Eq. (3) $(-)$ for L389 (middle curves) and H1080 21 (lower curves) at $130{ }^{\circ} \mathrm{C}$, and for $\mathrm{H} 984 \_57$ (upper curves) at $205^{\circ} \mathrm{C}$. Data for the latter were vertically shifted by a factor of two for clarity.

where $\dot{\gamma}$ is the shear rate. For linear polymers, this empirical rule often holds, although the dynamic viscosity can be somewhat larger than $\eta_{s s}(\dot{\gamma})$ [Booij et al. (1983); Bird et al. (1987); Venkatraman et al. (1990); Ferri and Lomellini (1999)].

In Fig. 1, dynamic and steady state viscosities are shown for a linear PIB, L389, and two hyperbranched samples, H1080_21 and H984_57, at the indicated temperatures. The data for H984_57 were shifted vertically by a factor of two for clarity, since at $205^{\circ} \mathrm{C}$ the viscosities are equal to those for $\mathrm{L} 389$ at $130^{\circ} \mathrm{C}$. This equivalence provides a comparative basis to be exploited below. As seen in Fig. 1, the Cox-Merz rule holds reasonably well for the branched PIB; that is, it is at least as accurate as for linear polymers. The small-strain oscillatory and steady state shear measures of viscosity agree to within $10 \%$ for all of the PIB materials. For the linear PIB, the dynamic viscosity is slightly higher than for steady shearing. This is not unusual, although the range of the steady state data is limited. From previous results for branched polyethylene [Schulken et al. (1980); Booij et al. (1983); Wood-Adams (2001)] and branched polystyrene [Ferri and Lomellini (1999)], it has been suggested that $\eta_{s s}(\dot{\gamma})>\eta^{*}(\omega)$. This trend is not evident in our data for the hyperbranched PIB.

Another empirical equation, suggested by Gleissle (1980), relates the steady state viscosity to the transient viscosity, $\eta^{+}$, measured during the approach to steady-state at the limiting shear rate $\dot{\gamma} \rightarrow 0$.

$$
\left.\lim _{\dot{\gamma} \rightarrow 0} \eta^{+}(t, \dot{\gamma})\right|_{t^{-1}=\dot{\gamma}}=\eta_{s s}(\dot{\gamma})
$$

The subscript denotes that the startup viscosity is taken in the Newtonian regime, at shearing times equal to the reciprocal of the corresponding shear rate. Good agreement with Eq. 3 has been reported for several polymers, including linear PIB [Gleissle (1980)] and branched polyethylene [Laun (1986); Wood-Adams (2001)]. 
TABLE II. Shearing thinning parameters.

\begin{tabular}{ccc}
\hline \hline Designation & $M_{w} M_{n}$ & $n$ [Eq. (4)] \\
\hline L176 & 1.6 & 0.19 \\
L302 & 1.8 & 0.23 \\
L389 & 2.5 & 0.47 \\
H984_57 & 1.3 & 0.46 \\
H230_9 & 2.1 & 0.59 \\
H1080_21 & 2.6 & 0.53 \\
\hline \hline
\end{tabular}

Included as the solid lines in Fig. 1 are the transient data, measured at $\dot{\gamma}$ $=10^{-3} \mathrm{~s}^{-1}$, which is quite close to the zero-shear-rate limit. For linear PIB, the $\eta^{+}(t)$ are equal within experimental error to the steady state viscosities, although the latter do not extend beyond $0.1 \mathrm{~s}^{-1}$. For the hyperbranched PIBs, $\eta^{+}(t)$ exceeds $\eta_{s s}$. At least for this material, the Gleissle relation is less accurate than the Cox-Merz rule.

\section{Shear rate dependence}

A primary objective of using hyperbranched polymers is to improve the processing. Long-chain branching has been reported to induce shear thinning at lower frequencies [Kim et al. (1996); Wood-Adams (2001)], although this is also affected by polydispersity [Bird et al. (1987)]. A shear thinning viscosity facilitates flow at the high deformation rates of practical importance. This property can be expressed as a power-law (Bird et al. 1987)

$$
\eta \propto \dot{\gamma}^{n-1},
$$

in which the parameter $n \leqslant 1$.

Results for linear and hyperbranched PIBs, obtained from the dynamic viscosity in the power law regime (i.e., at frequencies, $0.1 \leqslant \omega \leqslant 10 \mathrm{rad} / \mathrm{s}$, beyond the zero-shear rate regime), are listed in Table II. Although the exact value of $n$ depends on shear rate, some general observations can be made. Linear polyisobutylene has a value of $n$ that increases (less shear thinning) with increasing polydispersity. This effect of the molecular weight distribution on shear thinning is well known [Tokita and Pliskin (1973)], and complicates an assessment of the effect of branching. Nevertheless, the hyperbranched samples in Table II have large $n$, suggesting that hyperbranched PIBs tend to be less shear thinning than their linear counterparts. A similar result was reported by Simon et al. (2001) for highly branched poly(methyl methacrylates). It is also consistent with the broader relaxation spectrum of branched chains [see, for example Bero and Roland (1996)]. However, an inference from Table II is that by adjustment of polydispersity, similar shear rate dependencies can be obtained for the branched and linear polymers.

The shear thinning results in Table II were obtained from small-strain dynamic experiments; that is, we are relying on the Cox-Merz rule in assessing shear thinning behavior from linear viscoelastic data. However, branched and linear polymers have been shown to exhibit different strain dependences of their viscoelastic properties [Osaki and Kurata (1980); Bick and McLeish (1996)]. At high strains, Tokita and Pliskin (1973) and Jaćović et al. (1979) reported greater shear thinning for branched polymers. Accordingly, a reliable assessment of the processing of hyperbranched and linear PIB requires measurement of their nonlinear rheology, in combination with a meaningful basis for comparison.

Unlike most polymers with entangled branches, PIB exhibits thermorheological simplicity in the terminal zone [Santangelo et al. (1999); Robertson et al. (2001)]. In Fig. 2 


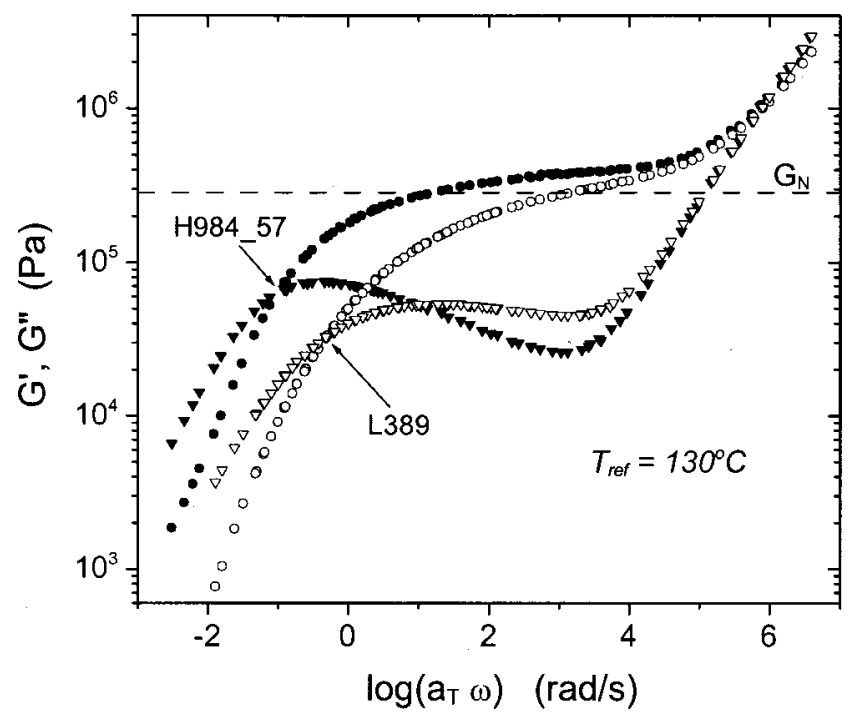

FIG. 2. Storage $(\bigcirc)$ and loss $(\nabla)$ moduli for H984_57 (solid symbols) and L389 (open symbols). The dashed line indicates the value of the plateau modulus, $G_{N}=290 \mathrm{kPa}$, for linear PIB reported by Santangelo et al. (1999).

are displayed master curves of the dynamic moduli of a hyperbranched PIB, H984_57, and a linear PIB, L389. At high frequencies ( $>10^{5} \mathrm{rad} / \mathrm{s}$ ), associated with the approach of the transition zone, the relaxation functions merge, since branching does not change the glass temperature. At lower frequencies, however, the curves diverge. The branched PIB exhibits a more developed rubbery plateau, attaining terminal behavior only at very low frequencies. Nevertheless, the slopes of the loss moduli in the terminal zone are quite similar for the two samples. This is consistent with the data in Table II, in that the polydispersity is much larger for L389 $\left(M_{w} / M_{n}=2.5\right)$ than for H984_57 $\left(M_{w} / M_{n}\right.$ $=1.3$. .

The similarity of their shear rate dependences suggests utilizing these two samples for a comparison of nonlinear rheological properties. However, as evident from Fig. 2, the respective viscosities are quite different. At the reference temperature for the data $\left(130^{\circ} \mathrm{C}\right)$, the zero shear viscosity of L389 is $2.58 \times 10^{5} \mathrm{~Pa} \mathrm{~s}$, a factor of 22 smaller than $\eta_{0}$ for H984_57. We can reduce the viscosity of H984_57 by increasing its temperature. For H984_57, $\eta_{0}$ at $205^{\circ} \mathrm{C}$ is 2.94E5 Pa s, which is within $10 \%$ of the value for L389 at $130{ }^{\circ} \mathrm{C}$. Moreover, the respective shear-rate dependences of the viscosities can be seen (Fig. 3) to remain very similar. Thus, a rational comparison of the processing behavior of the linear and branched PIB can be made from measurements at $130^{\circ} \mathrm{C}$ and $205^{\circ} \mathrm{C}$, respectively.

We note in passing that the factor of 22 decrease in viscosity of the hyperbranched PIB in going from $130{ }^{\circ} \mathrm{C}$ to $205^{\circ} \mathrm{C}$ is greater than the change in $\eta_{0}$ predicted by the VogelFulcher parameters reported previously [Robertson et al. (2001)]. However, the fitting to the Vogel-Fulcher therein was limited to temperatures not exceeding $150^{\circ} \mathrm{C}$. Obviously, there can be significant error associated with extrapolation beyond the measured range.

In Fig. 4 are displayed the corresponding values for the steady state primary normal stress difference, $N_{1}$. These data are shown as a function of shear stress [Han (1976)], although a similar plot results from the use of shear rate as the abscissa. Within the 


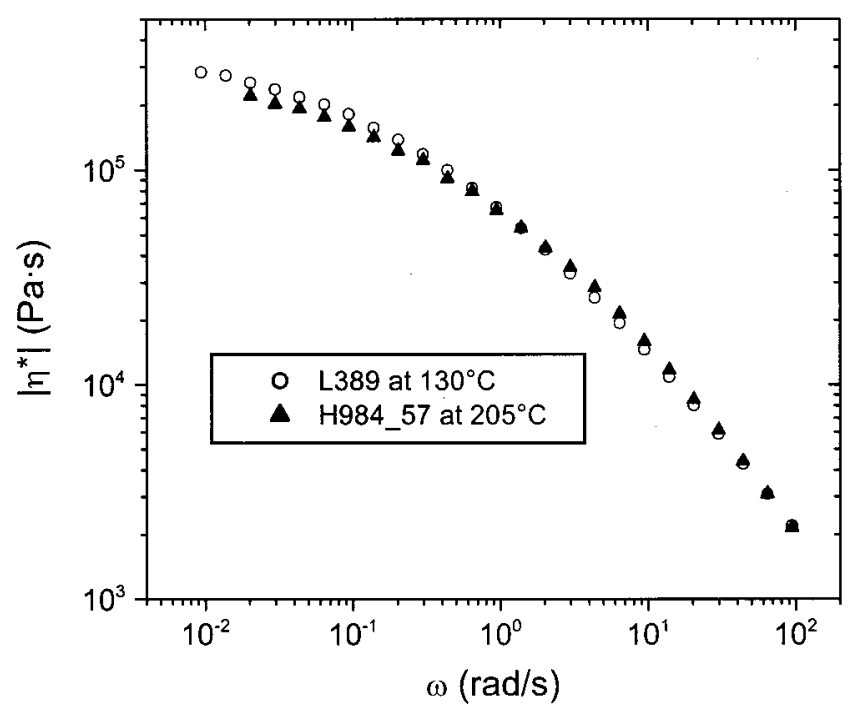

FIG. 3. Dynamic viscosities measured for L 389 at $130{ }^{\circ} \mathrm{C}$ and $\mathrm{H} 984 \_57$ at $205^{\circ} \mathrm{C}$ (creep viscosities are equivalent at these temperatures).

experimental uncertainty, this measure of elasticity is equal for H984_57 and L389, at least when compared at the respective temperatures for which their viscosities are equal.

\section{Transient shear flow}

Polymer melts and entangled solutions exhibit overshoots of the stress during the startup of shear flow. The origin of this transient response is considered to be the orientation and consequent decrease in the entanglement density of the chain molecules [Graessley (1965); Porter and Johnson (1966); Wagner and Meissner (1980); Isono et al.

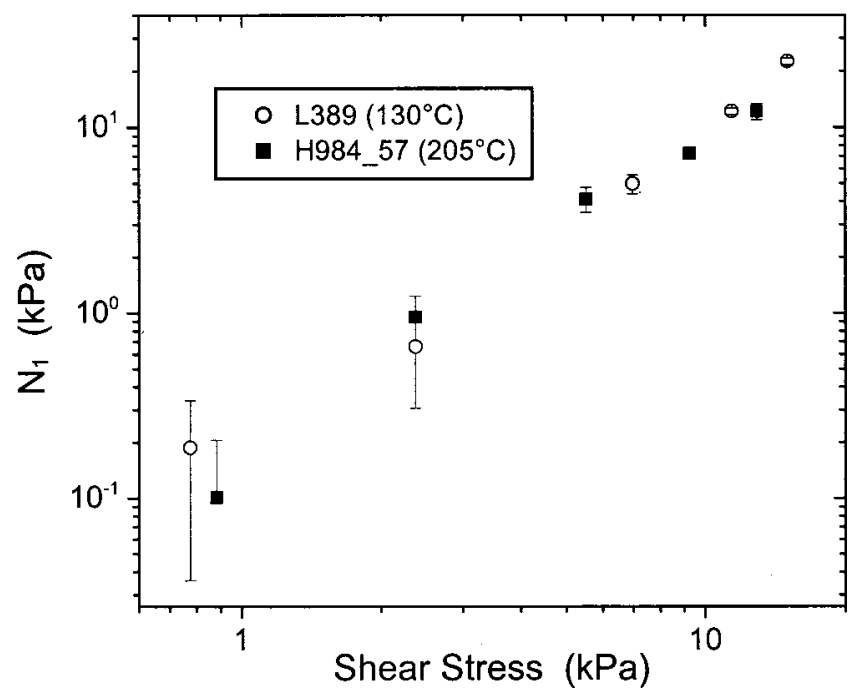

FIG. 4. Shear stress dependence of first normal stress difference for linear and hyperbranched PIB at respective temperatures for which creep viscosities are equal. The data were obtained over the frequency range 0.003 $\leqslant \dot{\gamma} \leqslant 0.1 \mathrm{~s}^{-1}$. Some of the error bars appear skewed because of the logarithmic ordinate scale. 


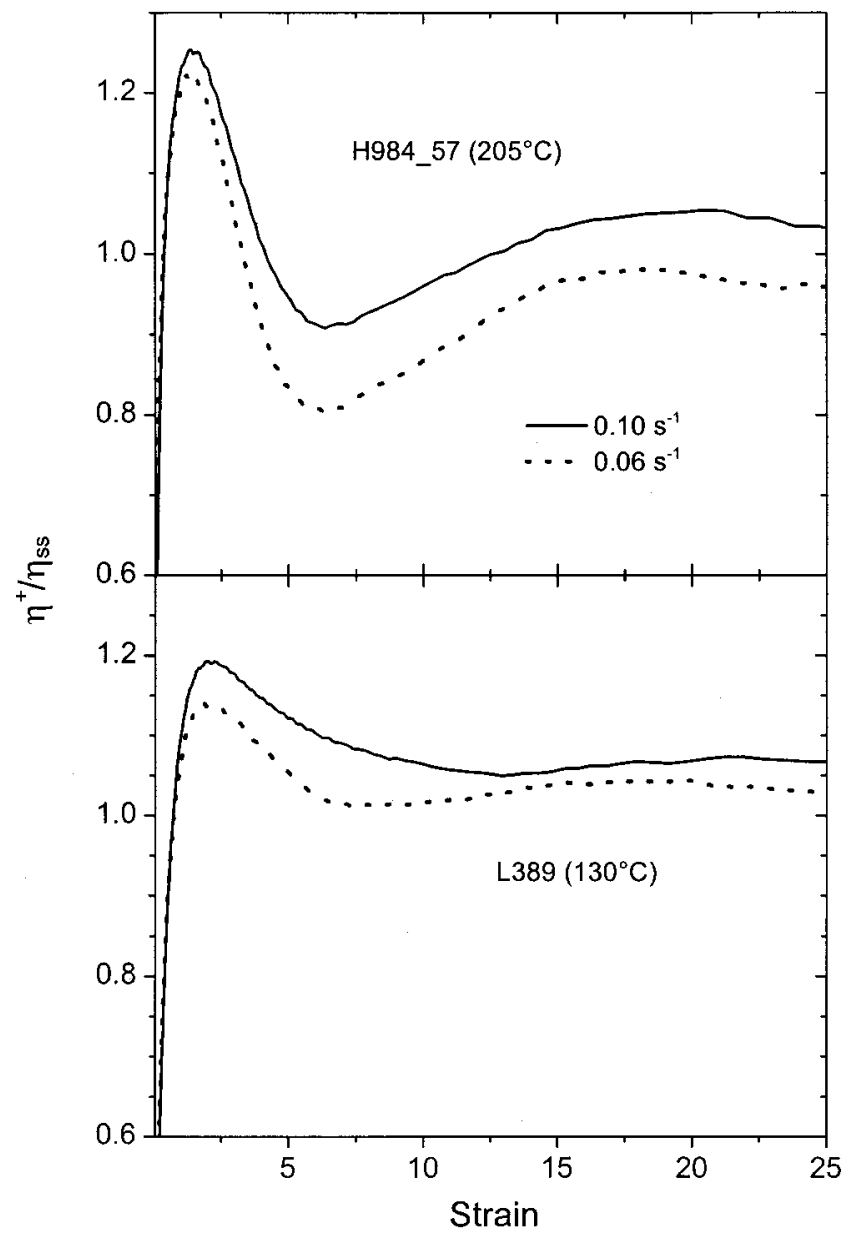

FIG. 5. Transient shear viscosities, normalized by the steady-state viscosity for linear and branched PIB at the indicated $\dot{\gamma}$. The abscissa is the product of the shear rate times the shearing time.

(1997); Oakley et al. (1998)], although unentangled polymers show similar effects [Santangelo and Roland (2001); Osaki et al. (2000)].

Figure 5 shows the transient shear viscosity, measured prior to attainment of steady state, for L389 and H984_57. For both samples, an overshoot is observed, roughly $20 \%$ larger than $\eta_{s s}$. However, at equal shear rate, the hyperbranched PIB exhibits a more prominent maximum in $\eta^{+}$. Overshoots in the first normal stress difference are also commonly observed during startup of shear flow. These were observed herein, but the scatter in the data was too large for any differences between the two PIB samples to be discerned.

As suggested by the longer time data in Fig. 5, H984_57 required a longer shearing time to reach steady state than did the linear PIB. The maximum in $\eta^{+}$for H984_57, however, transpired at shorter times, corresponding to shear strains $(=\dot{\gamma} \times t)$ equal to 1.5 \pm 0.2. This overshoot strain for L389 was $2.0 \pm 0.2$, which is within the range of literature values for entangled linear polymers [Bird et al. (1987)]. For both PIB, the strain associated with the maximum in the shear stress was independent of shear rate. 


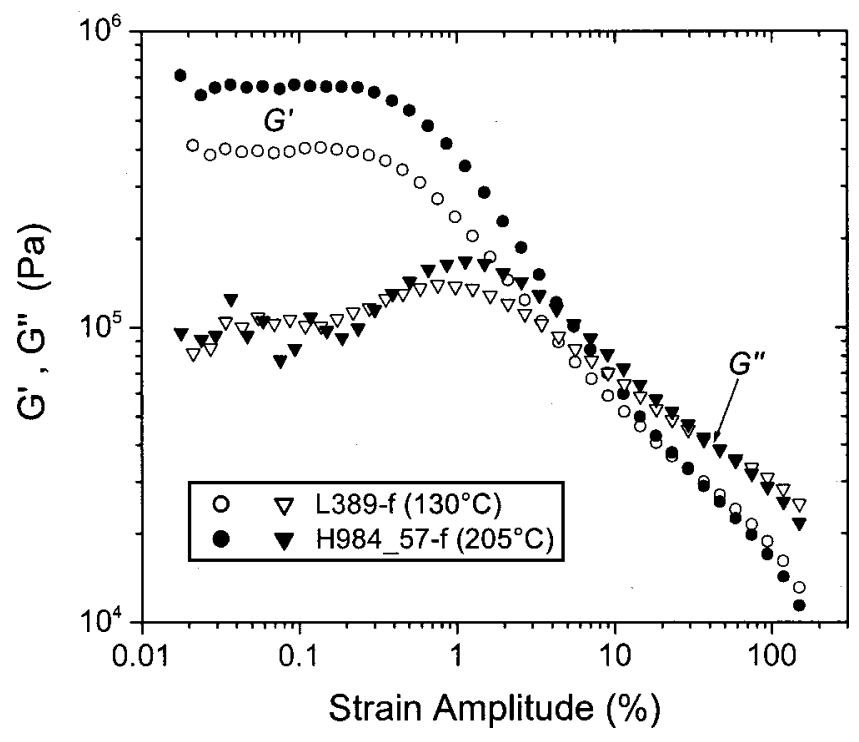

FIG. 6. Payne effect for the filled PIB compounds measured at $\omega=0.63 \mathrm{rad} / \mathrm{s}$.

\section{B. Filled compounds}

The comparisons made in Figs. 3-5 are limited to relatively low frequencies, while typical polymer processing operations involve very high deformation rates. Since the behavior of the two PIBs may diverge at higher frequencies, it is of interest to compare their rheology under such conditions. To do this we use capillary rheometry; with the available equipment, shear rates exceeding $10^{3} \mathrm{~s}^{-1}$ can be achieved. However, at even the lowest shear rates, smooth flows could not be obtained with either L389 or H984_57. The flow was unsteady and the extrudates exhibited substantial melt fracture. Raising the temperature to as high as $260^{\circ} \mathrm{C}$ for the hyperbranched PIB did not overcome this problem. Consequently, we make recourse to the usual solution to this problem in the rubber industry, to wit, incorporation of reinforcing filler (carbon black) into the polymers.

Compounds were prepared containing 100 parts of PIB, 50 parts N339 carbon black, and in addition, we added five parts paraffin oil, primarily to facilitate mixing, although it reduces the viscosity as well. In order to achieve good dispersion of the filler, the rubbers were milled following incorporation of the carbon black in the internal mixer. A measure of this dispersion is the strain amplitude dependence of the storage modulus. The filler network yields high modulus at low strains, which decreases at a critical strain associated with deflocculation of the carbon black agglomerates; this phenomenon is the well-known Payne effect [Payne (1964, 1965); Roland (1990)]. Figure 6 shows the dynamic moduli for filled compounds of the L389 and H984_57. At low strains, $G^{\prime}$ for the branched material is about $40 \%$ larger than $G^{\prime}$ for the linear PIB. This suggests greater filler agglomeration in the former, perhaps because the core region of the highly branched structure is less accessible to the filler particles.

At higher strains $(\geqslant 10 \%)$, after disruption of the filler network, the storage moduli for the two materials are quite comparable. This breakup of the flocculated structure gives rise to the maxima in the loss moduli seen in Fig. 6. The strain for the capillary flow experiments is certainly sufficient to disrupt the agglomerated filler. 


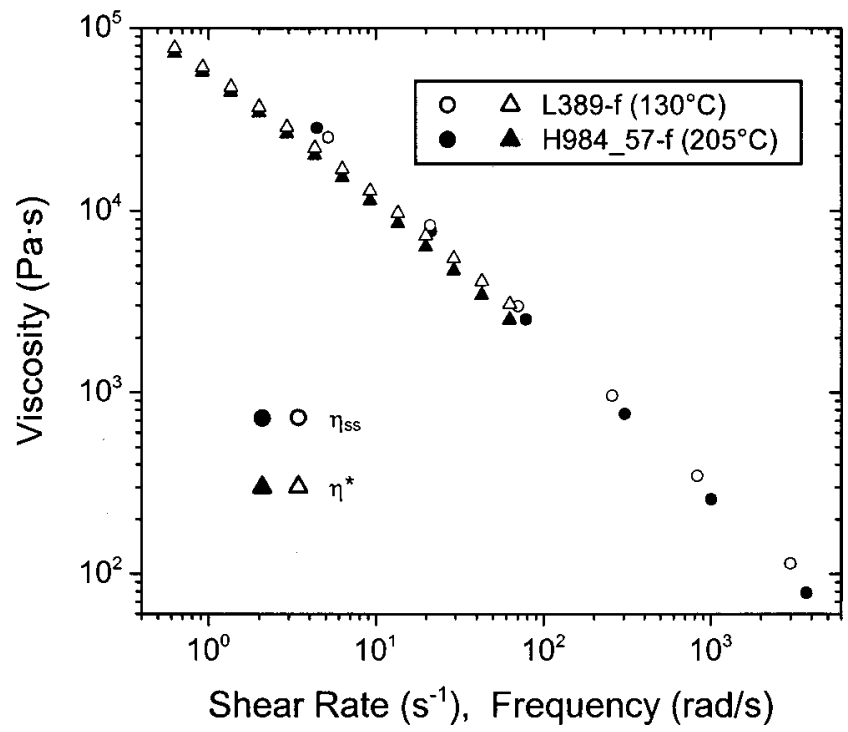

FIG. 7. Shear viscosity from capillary rheometry (circles) and the dynamic viscosity (triangles) for filled PIB. The dynamic mechanical data were obtained at a strain amplitude of $10 \%$, at which the filler agglomerate structure is absent.

In Fig. 7, we display the dynamic viscosity measured at $10 \%$ strain amplitude as a function of frequency. This strain is sufficiently high that the carbon black network is not contributing. Again, we carry out the measurements at temperatures, $130{ }^{\circ} \mathrm{C}$ and $205^{\circ} \mathrm{C}$, for which the neat polymers have equivalent viscosities. Included in Fig. 7 are the shear viscosities obtained from the capillary rheometry experiments. Rough conformance to the Cox-Merz rule [Eq. (2)] is observed. More significantly, the respective shear-rate dependences of the viscosity for the linear and branched PIB remain equal. This is expected to the extent there is similar interaction (primarily dispersion forces) of the filler with either polymer. Thus, the shear-thinning behavior observed in the low strain dynamic measurements on the neat polymers is retained in higher shear rate capillary flow experiments on the filled compounds.

At modest deformation rates, the elastic stresses were equivalent for the two neat PIB (Fig. 4). We cannot measure the secondary forces in the capillary rheometer. However, the magnitude of the elastic response is reflected in the pressure drop deduced from the Bagley analysis. These results are displayed in Fig. 8. At the lower shear stresses, the pressure drop is comparable for the two samples, consistent with Fig. 4. Only at shear rates $>10^{2} \mathrm{~s}^{-1}$ do the behaviors diverge, with the linear PIB apparently developing larger elastic stresses.

The measurement temperature for L 389 is $75^{\circ}$ degrees lower than that of the branched PIB. Although this yields equivalent viscosities, the temperature dependence of the elastic properties is unknown. However, there is no reason to believe it will differ from that of the viscosity, given the thermorheological simplicity of these materials [Santangelo et al. (1999); Robertson et al. (2001)]. Since the entrance pressure drop is a measure of the elongational viscosity, Figure 4 indicates that branching reduces the Trouton ratio in PIB. This is contrary to a recent suggestion of Larson (2001) that long-chain branching results in a larger Trouton ratio.

Estimates of the primary normal stress difference are sometimes attempted from pressure drop data [Carreau et al. (1997)]. The problems with such an analysis are well 


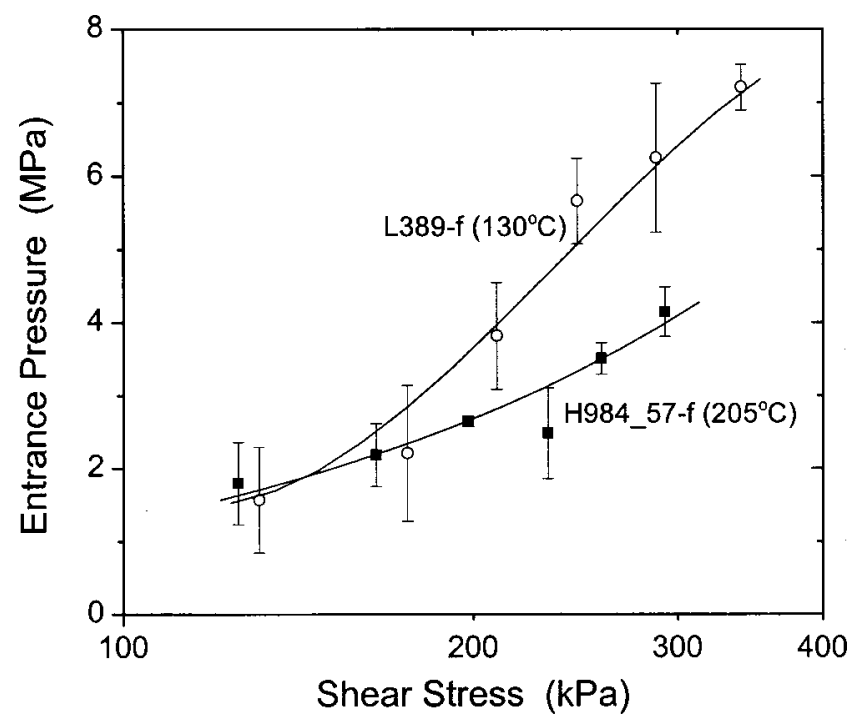

FIG. 8. Pressure drop associated with the capillary ends as deduced from the Bagley analysis, for filled PIB over the range $4<\dot{\gamma}<4000 \mathrm{~s}^{-1}$. The smooth lines are visual aides only.

documented [Choplin and Carreau (1981); Boger and Denn (1980)]. When $N_{1}$ is calculated from the entrance pressures in Fig. 8, the observed trends are unchanged.

The relaxation of the normal forces when the polymer exits the capillary gives rise to elastic recovery. From the data in Fig. 8, we expect that at higher shear stresses, the extrudate swell should be larger for the linear PIB than the hyperbranched sample. This is indeed the case, as shown in Fig. 9. These data, obtained nine days after the capillary rheometer experiments, represent the final values for the elastic recovery. In the smallstrain limit, this recoil strain is proportional to the recoverable compliance, $J_{e}$. However,

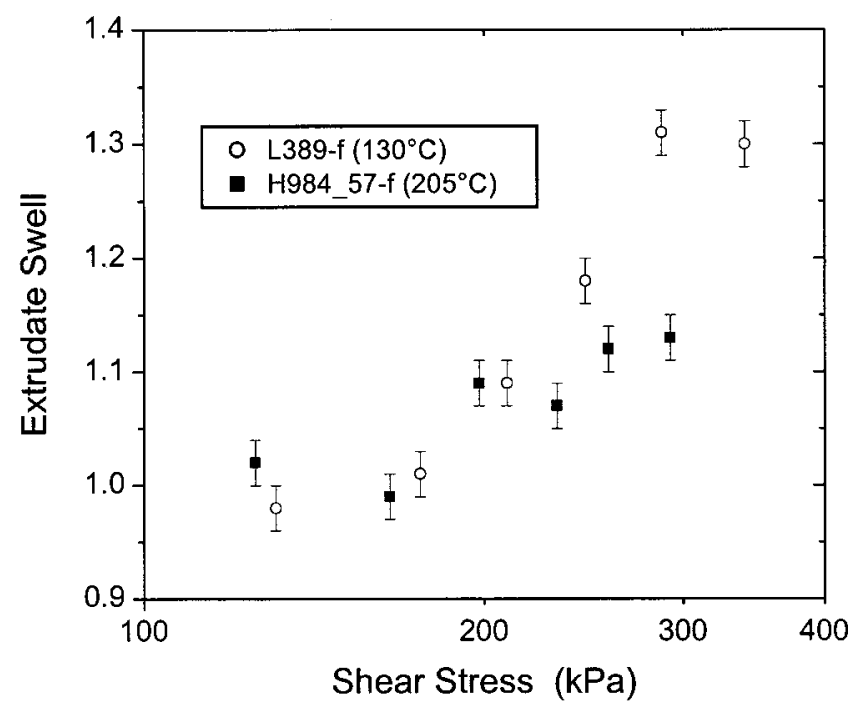

FIG. 9. Extrudate swell, defined as the ratio of the extrudate diameter to the capillary diameter, measured for the filled PIB, nine days at room temperature after extrusion through the die with an aspect ratio of 25 . 
polydispersity of molecular weight and long-chain branching both increase $J_{e}$ relative to the value for the linear polymer [Masuda et al. (1971); Plazek and Agarwal (1976); Rochefort et al. (1979)]. For the present materials, compared at equal viscosity, elastic effects appear to be larger for the linear PIB, at least at higher shear rates.

\section{CONCLUSIONS}

At moderate shear rates, a polydisperse $\left(M_{w} / M_{n}=2.5\right)$ linear PIB is shown to have very similar rheological properties to a hyperbranched PIB with narrower molecular weight distribution $\left(M_{w} / M_{n}=1.3\right)$. This determination was made by taking advantage of the fact that both linear and branched PIB are thermorheologically simple in the terminal zone. Among polymers with long-chain branching, polyisobutylene is unusual in this respect [Santangelo et al. (1999); Robertson et al. (2001)]. The thermorheological simplicity allows a comparison of processing behavior at respective temperatures for which their viscosities are equal. Under these conditions, the linear and hyperbranched PIB have equivalent shear-rate dependences of both their viscosity and normal stress. The transient responses were also similar, although H984_57 exhibited a slightly higher stress overshoot, and required a longer time to attain steady state. The implication is that branching per se may not confer unique behavior, at least in steady state shear experiments on neat polymers.

At lower shear rates, the similarities in flow properties were also observed in capillary rheometry measurements on the PIB reinforced with carbon black. However, limited results at high shear rates $\left(>100 \mathrm{~s}^{-1}\right)$ suggest greater elasticity in the linear PIB, leading to larger entrance pressures and extrudate swells. This aspect of the flow behavior deserves further examination.

\section{ACKNOWLEDGMENTS}

The work at NRL was supported by the Office of Naval Research. One of the authors (C.G.R.) expresses his gratitude for an ASEE-NRL postdoctoral fellowship. The authors thank Dr. C. Paulo for the synthesis of the polymers and N. Yue, who carried out gel permeation chromatography.

\section{References}

Atilgan, A. R., T. Haliloglu, I. Bahar, and B. Erman, "Correlated fluctuations in polymer networks," Comput. Theor. Polym. Sci. 8, 55-59 (1998).

Bagley, E. B., "End corrections in the capillary flow of polyethylene" J. Appl. Phys. 28, 624-627 (1957).

Bero, C. A. and C. M. Roland, "Terminal relaxations in linear and 3-arm star polyisoprenes," Macromolecules 29, 1562-1568 (1996).

Bick, D. K. and T. C. B. McLeish, "Topological contributions to nonlinear elasticity in branched polymers," Phys. Rev. Lett. 76, 2587-2590 (1996).

Bird, R. B., R. C. Armstrong, and O. Hassager, Dynamics of Polymeric Liquids, 2nd ed. (Wiley, New York, 1987), Vol. 1

Boger, D. V. and M. M. Denn, "Capillary and slit methods of normal stress measurements," J. Non-Newtonian Fluid Mech. 6, 163-185 (1980).

Booij, H. C., P. Leblans, J. Palmen, and G. Tiemersma-Thoone, "Nonlinear viscoelasticity and the Cox-Merz relations for polymeric fluids," J. Polym. Sci., Polym. Phys. Ed. 21, 1703-1711 (1983).

Carreau, P. J., D. C. R. De Kee, and R. P. Chabra, Rheology of Polymeric Systems (Hanser Verlag, New York, 1997).

Choplin, L. and P. J. Carreau, "Excess pressure losses in a slit," J. Non-Newtonian Fluid Mech. 9, 119-146 (1981). 
Cox, W. P. and E. H. Merz, "Correlation of dynamic and steady state flow viscosities," J. Polym. Sci. 28, 619-622 (1958).

Ferri, D. and P. Lomellini, "Melt rheology of randomly branched polystyrenes," J. Rheol. 43, 1355-1372 (1999).

Ferry, J. D., Viscoelastic Properties of Polymers, 3rd ed. (Wiley, New York, 1980).

Fetters, L. J., A. D. Kiss, D. S. Pearson, G. F. Quack, and F. J. Vitus, "Rheological behavior of star-shaped polymers," Macromolecules 26, 647-654 (1993).

Flory, P. J., "Effects of cross-linking and branching on the molecular constitution of diene polymers," J. Am. Chem. Soc. 69, 2893-2899 (1947).

Gleissle, W., in Rheology, edited by G. Astarita, G. Marucci, and L. Nicolais (Plenum, New York, 1980), Vol. 2, pp. 457-462.

Graessley, W. W., "Molecular entanglement theory of flow behavior in amorphous polymers," J. Chem. Phys. 43, 2696-2703 (1965).

Graessley, W. W., "Effect of long branches on the flow properties of polymers," Acc. Chem. Res. 10, 332-339 (1977).

Han, C. D., Rheology in Polymer Processing (Academic, New York, 1976).

Hingmann, R. and B. L. Marczinke, "Shear and elongational flow properties of polypropylene melts," J. Rheol. 38, 573-587 (1994).

Inkson, N. J., T. C. B. McLeish, O. G. Harlen, and D. J. Groves, "Predicting low density polyethylene melt rheology in elongational and shear flows with "pom-pom" constitutive equations," J. Rheol. 43, 873-896 (1999).

Isono, Y., T. Kamohara, A. Takano, and T. Kase, "Nonlinear viscoelastic properties and change in entanglement structure of linear polymer," Rheol. Acta 36, 245-251 (1997).

Jaćović, M. S., D. Pollock, and R. S. Porter, "A rheological study of long branching in polyethylene by blending," J. Appl. Polym. Sci. 23, 517-527 (1979).

Janzen, J. and R. H. Colby, "Diagnosing long-chain branching in polyethylenes," J. Mol. Struct. 486, 569-584 (1999).

Kasehagen, L. J. and C. W. Macosko, "Nonlinear shear and extensional rheology of long-chain randomly branched polybutadiene," J. Rheol. 42, 1303-1327 (1998).

Kennedy, J. P., L. R. Ross, and O. Nuyken, "New telechelic polymers and sequential copolymers by polyfunctional initiator-transfer agents (Inifers). 14. Characterization of linear and three-arm star polyisobutylenes by degradative oxidation," Polym. Bull. 5, 5-10 (1981).

Kim, Y. S., C. I. Chung, S. Y. Lai, and K. S. Hyun, "Melt rheological and thermodynamic properties of polyethylene homopolymers and poly(ethylene/ $\alpha$-olefin) copolymers with respect to molecular composition and structure," J. Appl. Polym. Sci. 59, 125-137 (1996).

Larson, R. G., "Combinatorial rheology of branched polymer melts," Macromolecules 34, 4556-4571 (2001).

Laun, H. M., "Prediction of elastic strains of polymer melts in shear and elongation," J. Rheol. 30, 459-501 (1986).

Masuda, T., Y. Ohta, and S. Onogi, "Rheological properties of anionic polystyrenes, III. Characterization and rheological properties of four-branch polystyrenes," Macromolecules 4, 763-768 (1971).

Meissner, J., "Basic parameters, melt rheology, processing and end-use properties of three similar low density polyethylene samples," Pure Appl. Chem. 42, 553-612 (1975).

Oakley, J. G., A. J. Giacomin, and J. A. Yosick, "Molecular origins of nonlinear viscoelasticity," Mikrochim. Acta 130, 1-28 (1998).

Osaki, K. and M. Kurata, "Experimental appraisal of the Doi-Edwards theory for polymer rheology based on the data for polystyrene solutions," Macromolecules 13, 671-676 (1980).

Osaki, K., T. Inoue, and T. Isomura, "Stress overshoot of polymer solutions at high rates of shear," J. Polym. Sci., Polym. Phys. Ed. 38, 1917-1925 (2000).

Paulo, C. and J. E. Puskas, "Synthesis and characterization of hyperbranched polyisobutylenes," Macromolecules 34, 734-739 (2001).

Payne, A. R., "The role of hysteresis in polymers," Rubber J., 146, 36-49 (1964).

Payne, A. R., in Reinforcement of Elastomers, edited by G. Kraus (Interscience, New York, 1965), Chap. 3.

Pearson, D. S., S. J. Mueller, L. J. Fetters, and N. Hadjichristidis, "Comparison of the rheological properties of linear and star-branched polyisoprenes in shear and elongational flows," J. Polym. Sci., Polym. Phys. Ed. 21, 2287-2298 (1983).

Plazek, D. J. and P. K. Agarwal, "The creep behavior of some commercial polystyrenes," Proc.-Int. 7th Congr. Rheol. (1976), pp. 488-489.

Porter, R. S. and J. F. Johnson, “The entanglement concept in polymer systems," Chem. Rev. 66, 1-27 (1966).

Robertson, C. G., C. M. Roland, C. Paulo, and J. E. Puskas, "Linear viscoelastic properties of hyperbranched polyisobutylene," J. Rheol. 45, 759-772 (2001).

Rochefort, W. E., G. G. Smith, H. Rachapudy, V. R. Raju, and W. W. Graessley, "Properties of amorphous and crystallizable hydrocarbon polymers. II. Rheology of linear and star-branched polybutadiene," J. Polym. Sci., Polym. Phys. Ed. 17, 1197-1210 (1979). 
Roland, C. M., "Dynamic mechanical behavior of filled rubber at small strains," J. Rheol. 34, 25-34 (1990). Santangelo, P. G., C. M. Roland, and J. E. Puskas, "Rheology of star-branched polyisobutylene," Macromolecules 32, 1972-1977 (1999).

Santangelo, P. G. and C. M. Roland, "Interrupted shear flow of unentangled polystyrene melts," J. Rheol. 45, 583-594 (2001).

Schulken, R. M., R. H. Cox, and L. A. Minnick, "Dynamic and steady state rheological measurements on polymer melts,” J. Appl. Polym. Sci. 25, 1341-1353 (1980).

Simon, P. F. W., A. H. E. Muller, and T. Pakula, "Charaterization of highly branched poly(methylmethacrylate) by solution viscosity and viscoelastic spectroscopy," Macromolecules 34, 1677-1684 (2001).

Tokita, N. and I. Pliskin, "The dependence of processability on the molecular weight distribution of elastomers," Rubber Chem. Technol. 46, 1166-1187 (1973).

Venkatraman, S., M. Okano, and A. Nixon, "A comparison of torsional and capillary rheometry for polymer melts: The Cox-Merz rule revisited," Polym. Eng. Sci. 30, 308-313 (1990).

Wagner, M. H. and H. Meissner, "Network disentanglement and time-dependent flow behavior of polymer melts," Makromol. Chem. 181, 1533-1550 (1980).

Wood-Adams, P. M., "The effect of long-chain branches on the shear flow behavior of polyethylene," J. Rheol. 45, 203-210 (2001). 